\title{
Mycobacterial Lymphadenitis in Childhood
}

\author{
A. MACKELLAR, H. B. HILTON, and P. L. MASTERS \\ From Princess Margaret Hospital for Children, Perth, Western Australia
}

In Western Australia, as in many other countries, there has been in recent years a change in the ascribed aetiology of tuberculosis infection. We have gradually come to realize the importance of the role of anonymous mycobacteria in the production of tuberculous lesions in the superficial lymph nodes of children. We cannot say whether or not these infections occurred before our present study, but we intend to show that since 1959 the great majority of infections with a tuberculous histology have proved to be due not to the human or bovine tubercle bacillus, but to one or other of the anonymous group of mycobacteria.

The majority of these anonymous bacteria have been found to be resistant to antituberculous drugs in standard concentrations, so that many children might be saved the administration of potentially dangerous drugs if a reliable and rapid means of distinguishing between human and anonymous infection were available. Using routine bacteriological investigations the value of antituberculous drug therapy cannot be assessed until the results of culture and sensitivity tests are available, but this may take up to three months. Frequently no growth is obtained, and the clinician then has no bacteriological evidence on which to base a decision whether or not to continue drug therapy. Where there is evidence of active tuberculosis elsewhere in the body the decision will be simple, but where the disease is limited to one group of glands it will not be so easy.

For the past three years we have been helped in making the distinction between human and anonymous mycobacterial infections by the routine use of a double Mantoux test (Masters and Smyth, 1965). By using the test retrospectively we have been able to determine how many of our children presenting with superficial mycobacterial lymphadenitis during the past seven years were infected with anonymous strains.

We present the clinical, pathological, and epidemiological features of these infections, and the

Received May 16, 1966. results of treatment. The management is discussed in the light of our bacteriological findings.

\section{Material and Methods}

Between 1958 and the end of 196432 children were seen whose lymph nodes showed tuberculosis histology. In 30, acid-fast bacilli were identified either in pus or in sections, and those 30 children were included in this study.

Age and sex incidence (Table I). All the children were over 1 and less than 8 years, and all but four were under 5 years. There were 12 boys and 18 girls.

TABLE I

Age and Sex Incidence

\begin{tabular}{cc|c|c|c|c|c|c|c|c|c}
\hline & & $<1$ & $1+$ & $2+$ & $3+$ & $4+$ & $5+$ & $6+$ & $7+$ & \\
\hline Boys &. & 0 & 4 & 4 & 1 & 0 & 0 & 2 & 1 & 12 \\
\hline Tirls &. & 0 & 5 & 6 & 5 & 1 & 0 & 1 & 0 & 18 \\
\hline Total &.. & 0 & 9 & 10 & 6 & 1 & 0 & 3 & 1 & 30 \\
\hline
\end{tabular}

Duration of swelling. The average duration of swelling before presentation at the hospital was 6 weeks. In 15 children, or half of the series, the swelling had been present for less than a month, while in 2 it had been noted for less than one week. These 2 children and 3 others of this group presented with acute abscesses in the neck. In only one child had the swelling been present for more than 6 months.

Despite the short history, no fewer than 9 children in the whole series had abscesses or sinuses at the time of presentation.

Site of infection. Table II shows the various lymph nodes involved and the number of children developing abscesses in each site. It was often difficult to determine from the case records exactly which group or groups of glands were involved, and for this reason the group of upper cervical infections includes all those glands described as being below and behind the angle of the mandible. 6 children had two separate groups of glands involved, and 1 child had three separate groups with signs of disease. 
TABLE II

Site of Lymphadenitis and Abscess Formation

\begin{tabular}{|c|c|c|c|c|}
\hline \multicolumn{3}{|c|}{ Site } & No. & $\begin{array}{c}\text { No. With } \\
\text { Abscess }\end{array}$ \\
\hline $\begin{array}{l}\text { Facial ... } \\
\text { Submandibular } \\
\text { Upper cervical } \\
\text { Lower cervical } \\
\text { Epitrochlear } \\
\text { Inguinal }\end{array}$ & $\begin{array}{l}\ldots \\
\because \\
\cdots \\
\cdots\end{array}$ & $\begin{array}{l}\cdots \\
\cdots \\
\cdots \\
\cdots \\
.\end{array}$ & $\begin{array}{r}7 \\
12 \\
14 \\
2 \\
1 \\
3\end{array}$ & $\begin{array}{l}3 \\
3 \\
2 \\
1 \\
0 \\
0\end{array}$ \\
\hline Total .. & $\ldots$ & .. & $38^{\star}$ & 9 \\
\hline
\end{tabular}

*6 patients had lymphadenitis in 2 sites; 1 patient had lymphadenitis in 3 sites.

Mantoux testing. Antigens for the double Mantoux tests were supplied by the U.S. Public Health Service through the courtesy of Dr. Lydia Edwards. PPD-S is a purified protein derivative prepared by Dr. Florence Seibert (Seibert and Glenn, 1941), and has been adopted by the World Health Organisation as the international standard for purified protein derivative of mammalian tuberculin.

PPD-B is prepared from Battey bacilli (belonging to Runyon's Group III of anonymous mycobacteria), and before delivery to us was diluted to correspond in protein nitrogen content with PPD-S (Affronti, 1959). On this basis $0.1 \mathrm{ml}$. antigen corresponds to 5 tuberculin units.

Mantoux tests were performed by injecting $0.1 \mathrm{ml}$. of each antigen intradermally into the flexor aspect of the forearm using tuberculin syringes with No. 26 gauge needles. The test was read 2 or 3 days later by noting the average of the maximum and minimum diameters in millimetres of the area of induration at the site of the injection. The area of redness was not recorded, though it usually coincided with the area of induration.

Our first 15 children were tested with tuberculin purified protein derivative or PPD-S (100 tuberculin units, or $100 \mathrm{TU}$ ) obtained from the Commonwealth Serum Laboratories, Melbourne, and the results are shown in Fig. 1. 4 failed to react, or showed reactions of less than $5 \mathrm{~mm}$. in diameter: these were retested with PPD-S (1,000 TU) and 1 failed to react. Most of the positive reactions were weak ( 5 to $9 \mathrm{~mm}$. in diameter), and only 1 was strong (over $25 \mathrm{~mm}$.).

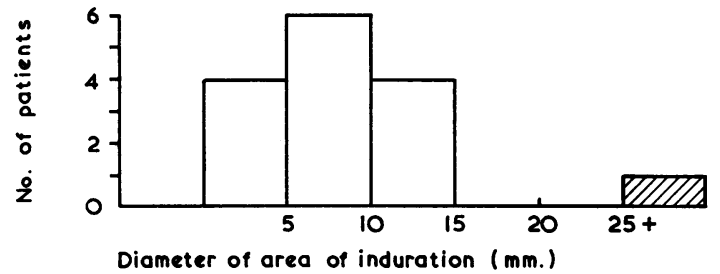

FIG. 1.-Standard Mantoux: PPD-S (100 TU), tested on preparation. Histogram showing number of patients and diameter of area of induration. The case represented by the hatched column in this and succeeding figures was considered to be infected by $M$. tuberculosis.

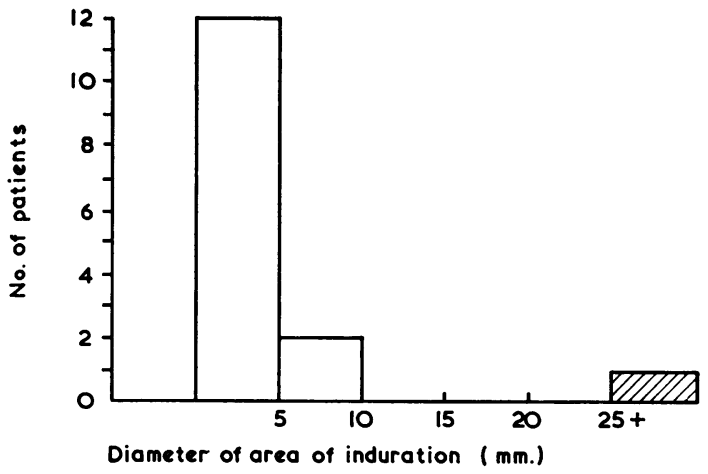

Fig. 2.-Double Mantoux: PPD-S (5 TU), tested retrospectively. Histogram, showing number of patients and diameter of area of induration.

These 15 children were later given double Mantoux tests, consisting of $5 \mathrm{TU}$ of PPD-S and the equivalent dose of PPD-B injected in opposite forearms. In 13 the test was given between 1 and 5 years after the onset of the lymphadenitis, and in 2 the interval was shorter. The reactions produced by $5 \mathrm{TU}$ of PPD-S are shown in Fig. 2. Most of the children showing weak reactions to the 100 TU of PPD-S failed to react to the smaller dose, but the child who developed the strong reaction to the large dose also reacted strongly to the small dose. In contrast, Fig. 3 shows the reactions produced by 5 TU of PPD-B. The children showing weak reactions to 100 TU of PPD-S reacted more strongly to the smaller dose of PPD-B, while the child (hatched columns in Fig. 1-3) who developed a strong reaction to the PPD-S extracts reacted less strongly to the PPD-B. We believe that this girl was infected by $M$. tuberculosis and that the other children were infected with anonymous mycobacteria.

The remaining 15 children were given double Mantoux tests on presentation (Table III). One child showed a bigger reaction to PPD-S than to PPD-B. The other 14 showed a bigger reaction to PPD-B, either initially or on repeating the test later. Combining the double Mantoux tests performed on presentation and retrospectively (Table III), only 2 out of 30 children showed a bigger reaction to PPD-S than to PPD-B.

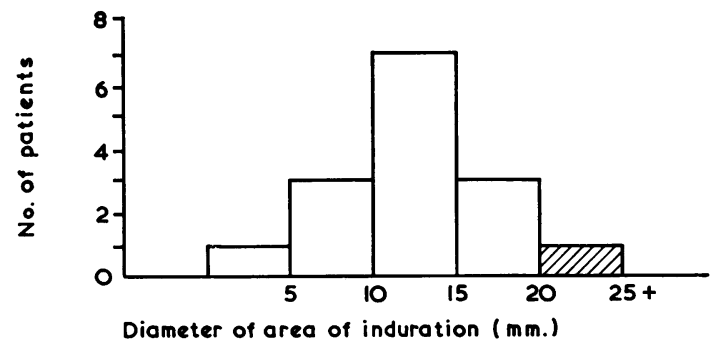

FIg. 3.-Double Mantoux: PPD-B (5 TU), tested retrospectively. Histogram, showing number of patients and diameter of area of induration. 
TABLE III

Results of Double Mantoux Tests

\begin{tabular}{|c|c|c|c|c|c|}
\hline & & \multicolumn{3}{|c|}{ Relative Sizes of Reactions } & \multirow{2}{*}{ Total } \\
\hline & & B $>$ S & $\mathbf{B}=\mathbf{S}$ & $S>B$ & \\
\hline $\begin{array}{l}\text { On presentation } \\
\text { Retrospective }\end{array}$ & $\therefore$ & $\begin{array}{l}11 \\
14\end{array}$ & $\begin{array}{l}3 \star \\
0\end{array}$ & $\begin{array}{l}1 \\
1\end{array}$ & $\begin{array}{l}15 \\
15\end{array}$ \\
\hline Total & . & 25 & 3 & 2 & 30 \\
\hline
\end{tabular}

* All 3 patients showed $B>S$ on repeating the double Mantoux test 8,2 , and 2 months later.

\section{Results}

Culture and sensitivity tests. Table IV correlates the results of the double Mantoux tests with cultural findings. All 11 children from whom anonymous strains of mycobacteria were grown showed a bigger reaction to PPD-B than to PPD-S, though in 2 children the reactions were equal on the first test. Similarly, both children from whom M. tuberculosis was grown showed a bigger reaction to PPD-S. Approximately half our cultures were sterile.

Table V shows the results of sensitivity tests carried out on the 13 mycobacteria grown from children in our series, together with 3 strains of M. tuberculosis grown from children with meningitis or primary complex in the lung.

All 11 anonymous strains were resistant to isoniazid and PAS, and 9 of them were resistant to streptomycin. In contrast, all 5 strains of $M$. tuberculosis were sensitive to streptomycin, isoniazid, and PAS.

Pathology. The sections of one or more lymph nodes from 29 patients were available for examination. Although the general histological appearances were similar to those due to $M$. tuberculosis infection, we found that giant cells were absent or extremely scanty in 14 out of 29 cases, and that large amounts of basophilic nuclear debris in areas of caseation were present in 5 of the 29 cases. These two histological criteria have been considered to
TABLE IV

Correlation of Double Mantoux Tests and Cultural Findings

\begin{tabular}{|c|c|c|c|}
\hline \multicolumn{2}{|l|}{ Mantoux } & \multicolumn{2}{|l|}{ Culture } \\
\hline Battey $>$ Standard initially or later & 28 & $\begin{array}{l}\text { Myco. Group III } \\
\text { Myco. Group II } \\
\text { No growth } \\
\text { Not cultured }\end{array}$ & $\begin{array}{r}8 \\
3 \\
13 \\
4\end{array}$ \\
\hline Standard > Battey & $3 t$ & $\begin{array}{l}\text { Myco. tuberculosis } \\
\text { No growth }{ }^{\star}\end{array}$ & $2 \dagger$ \\
\hline
\end{tabular}

${ }^{\star}$ Incubator overheated.

t Includes one child with primary pulmonary complex outside the series.

represent 'atypical' histology of tuberculous lymphadenitis by Jones and Campbell (1962).

Treatment. These children have been cared for by paediatricians and surgeons whose views on treatment sometimes differed. In the management of the 4 children presenting early in the series, before the nature of the infection was understood, treatment was begun on conservative lines with drug therapy alone. None of these children showed improvement after 2 to 6 months of apparently adequate therapy, and 2 progressed to abscess formation while under treatment. All 4 were ultimately submitted to operation, and retrospective Mantoux testing showed that their infections were of the anonymous variety.

In 9 children incision and drainage of an abscess was the primary treatment. All these later required excision of the gland or group of glands involved.

One child had incision and curettage of the lesion. The sinus healed and no further operation was required. This child was one of two with human tuberculous infection, and received drug therapy. Another child was submitted to tonsillectomy. In the remaining children excision of the involved glands was carried out, drug therapy being given to all but 8 .

The 4 children who were initially managed conservatively have already been discussed. Of the

TABLE V

Sensitivities of Mycobacteria Cultured

\begin{tabular}{|c|c|c|c|c|c|c|c|c|c|c|c|c|c|c|c|c|c|c|}
\hline \multirow{3}{*}{\multicolumn{2}{|c|}{ Organism }} & & \multicolumn{15}{|c|}{ Minimum Inhibitory Concentrations ( $\mu \mathrm{g} . / \mathrm{ml}$.) } & \multirow{3}{*}{$\begin{array}{c}\text { Total } \\
\text { Number } \\
\text { of Strains }\end{array}$} \\
\hline & & & \multicolumn{5}{|c|}{ Streptomycin } & \multicolumn{5}{|c|}{ Isoniazid } & \multicolumn{5}{|c|}{ PAS } & \\
\hline & & & 2 & 4 & 8 & 16 & $>16$ & $<0.1$ & $0 \cdot 1$ & $0 \cdot 2$ & $1 \cdot 0$ & $>1 \cdot 0$ & $<2$ & 2 & 4 & 8 & $>8$ & \\
\hline M. tuberculosis & . & $\ldots$ & 3 & 1 & 1 & & & 2 & & 3 & & & 3 & 2 & & & & $5^{\star}$ \\
\hline $\begin{array}{l}\text { Group II } \\
\text { Group III }\end{array}$ & 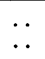 & 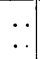 & & 1 & 1 & $\begin{array}{l}2 \\
1\end{array}$ & $\begin{array}{l}1 \\
5\end{array}$ & 1 & & & & $\begin{array}{l}3 \\
8\end{array}$ & & & & & $\begin{array}{l}3 \\
8\end{array}$ & $\begin{array}{l}3 \\
8\end{array}$ \\
\hline
\end{tabular}

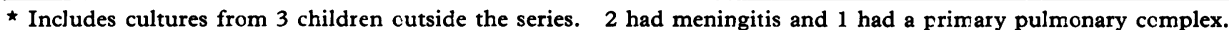


22 children treated primarily or secondarily by excision and drug therapy, 20 were cured and there were 2 relapses. There were 8 children treated by excision without drug therapy, of whom 7 were cured and one relapsed. The 3 children who suffered relapses all had infections with anonymous mycobacteria. In one the relapse took place in the same group of glands and was due to inadequate surgical excision. This child received no drug therapy. The relapses in the other 2 children occurred 4 and 12 months, respectively, after the initial infection, and they involved a different group of glands; in one child an infection involving the other side of the neck, and in the second an infection in the epitrochlear gland followed an infection in the cervical glands. Both these children had received antituberculous drug therapy from the time of their first operation until relapse took place. All 3 children who had suffered relapses were submitted to further surgical excision without drug therapy, and all were cured.

During the follow-up period 2 of our patients developed lymphadenopathy elsewhere in the neck, and these enlarged glands were removed for histological examination, but showed no evidence of mycobacterial infection.

Unpleasant sequelae of operations for tuberculous glands in the neck have been described on several occasions. None of our patients who have been followed for between 5 months and 4 years have unsightly scars, and none have residual nerve lesions, though 5 patients showed temporary facial weakness following operation.

\section{Evidence of Mycobacterial Infection in Other Members of the Family}

Patients infected by $M$. tuberculosis. In one child a history of tuberculous infection in another member of the family was obtained. The other child was an aboriginal foundling, so that no family history was available.

Patients with anonymous infections. The other 28 children are believed to have been infected with anonymous mycobacteria. Apart from 2 sisters who developed superficial lymphadenitisone beneath the mastoid and the other in the groinwithin 2 months of each other, no family or other contacts with active mycobacterial infection were detected. Patients were notified to the Tuberculosis Control Branch of the Department of Public Health, and chest radiographs of parents and other members of the family were taken where appropriate. Mantoux tests were made on members of 14 families. All sibs tested were negative, 7 to 1 in 1000 Old Tuberculin and 22 to the double Mantoux test. In one family both parents reacted to PPD-B and the father produced a smaller reaction to PPD-S. 3 fathers reacted to both PPDs, but the reaction to PPD-S was the greater. 4 mothers failed to react to either PPD.

In summary there was evidence of clinically inapparent family infection with anonymous mycobacteria in only one family, and no evidence of human-to-human spread in any family.

\section{Discussion}

Superficial mycobacterial lymphadenitis in Western Australian children is now usually due to infection by anonymous strains of mycobacteria. Others have found that these organisms commonly cause this infection in Canada, the United States, England, and Victoria, Australia (Masters and Smyth, 1965). Some of these reports show a higher incidence of infection by $M$. tuberculosis or a predominant anonymous strain other than the Battey bacillus. Jones and Campbell (1962) in Victoria reviewed 122 children with mycobacterial cervical lymphadenitis. Cultures were taken from 76 , of which 12 yielded $M$. tuberculosis and 15 yielded anonymous strains. Of these 15,13 were considered to be scotochromogens (Runyon's Group II). This contrasts with the predominance of Battey strains (Runyon's Group III) in our series.

The value of the double Mantoux test as a routine method of screening for mycobacterial infection and for distinguishing infections with $M$. tuberculosis from those caused by anonymous mycobacteria has been discussed by Masters and Smyth (1965). We are continuing to use the test routinely and would emphasize the value of repeating it when the reactions to the two antigens are of equal size or when only a weak reaction is produced. An increase in the reaction is valuable evidence of active infection, and we have found that when the reactions are nearly equal in size repetition of the test after a few weeks usually shows a significant difference between the reactions, indicating the nature of the infecting mycobacterium.

Of our 30 children, 26 were under 5 years, and a similar age incidence has been reported by Weed, Keith, and Needham (1956), Davis and Comstock (1961), and Black and Chapman (1964). In contrast, the series reported by Jones and Campbell (1962) included only 39 under 5 years out of 122 children.

Rapid development of the swelling and the formation of an abscess or sinus were frequent in our series. Jones and Campbell (1962) remarked on the 
shorter average duration of the swelling at presentation when anonymous mycobacteria were grown, compared with the duration when $M$. tuberculosis was grown.

The distinctive epidemiological features of anonymous mycobacterial infections are apparent in our series. These are the rare occurrence of man to man spread or clinical evidence of blood-stream spread in the body (Corpe, Runyon, and Lester, 1963).

We consider that the finding of acid-fast bacilli in smears of pus and in biopsies from superficial lymphadenitis is the most useful criterion of mycobacterial infection. The histological findings have been less helpful, e.g. in the differential diagnosis from catscratch disease (Jones and Campbell, 1962).

Our management of superficial mycobacterial lymphadenitis is based on three facts: (a) our ability to distinguish most infections due to anonymous strains by means of the double Mantoux test, ${ }^{\star}$ (b) the knowledge that most anonymous strains are resistant to the commonly used anti-tuberculous drugs (Wolinsky, 1960), and (c) the rarity of blood-stream spread due to these strains. It is now our practice not to give antituberculous drugs where the double Mantoux test clearly indicates an infection by an anonymous strain. We excise the whole group or groups of nodes involved and thus avoid recurrence and sinus formation.

\section{Summary}

The clinical, pathological, and epidemiological features of 30 children presenting with superficial mycobacterial lymphadenitis over a period of 7 years in Western Australia are described.

A double Mantoux test, consisting of 5 tuberculin units of PPD-S and PPD-B, was used retrospectively on 15 of the children and on presentation of the other 15 children. The results provide evidence that 28 of the children were infected by anonymous strains of mycobacteria and only 2 by $M$. tuberculosis.

\footnotetext{
* Since completing this paper, a further 4 cases with positive Mantoux test have been seen, 3 with Battey reaction greater than Standard, and from these 3 cases anonymous strains of mycobacteria were cultured. Our total series thus comprise 17 cases in which culture has been successful, and in all 17 cases culture and skin testing have correlated.
}

Twenty-six of our children were under 5 years of age and the average duration of the swelling on presentation was only 6 weeks. Abscesses or sinuses developed in 9 children.

The management of superficial mycobacterial lymphadenitis is discussed. When the double Mantoux test clearly indicates infection by anonymous mycobacteria, primary radical excision of the affected group of nodes without chemotherapy is suggested, as experience in Western Australia indicates that the anonymous strains are insensitive to antituberculous drugs and metastatic spread of the infection has not so far occurred.

None of the 28 children believed to have had anonymous mycobacterial infections had a family contact. In contrast, of the 2 infected by $M$. tuberculosis one had a family contact and the other was a foundling.

We are grateful to Dr. John Smyth for introducing the technique of the double Mantoux test to us, and to Dr. Lydia Edwards and the United States Public Health Service, for supplying the purified protein derivatives.

We wish to thank the Surgeons and Paediatricians of Princess Margaret Hospital for giving us access to the case notes of children under their care.

\section{REFERENCES}

Affronti, L. F. (1959). Purified protein derivatives (PPD) and other antigens prepared from atypical acid-fast bacilli and Nocardia asteroides. Amer. Rev. Tuberc., 79, 284.

Black, B. G., and Chapman, J. S. (1964). Cervical adenitis in children due to human and unclassified mycobacteria. Pediatrics, 33, 887.

Corpe, R. F., Runyon, E. H., and Lester, W. (1963). Status of disease due to unclassified mycobacteria. A statement of the subcommittee on unclassified mycobacteria of the Committee on Therapy. Amer. Rev. resp. Dis., 87, 459.

Davis, S. D., and Comstock, G. W. (1961). Mycobacterial cervical adenitis in children. F. Pediat., 58, 771.

Jones, P. G., and Campbell, P. E. (1962). Tuberculous lymphadenitis in childhood: the significance of anonymous mycobacteria. Brit. F. Surg., 50, 302.

Masters, P. L., and Smyth, J. T. (1965). A double mantoux test applied to screening children for mycobacterial infections: its value in distinguishing infections by anonymous strains. Aust.paediat. F., 1, 166.

Seibert, F. B., and Glenn, J. T. (1941). Tuberculin purified protein derivative; preparation and analyses of large quantity for standard. Amer. Rev. Tuberc., 44, 9.

Weed, L. A., Keith, H. M., and Needham, G. M. (1956). Nontuberculous acid-fast cervical adenitis in children. Froc. Mayo Clin., 31, 259.

Wolinsky, E. (1960). Experimental chemotherapy of atypical mycobacterial infection. In The Anonymous Mycobacteria in Human Disease, ed. J. S. Chapman, p. 132. C. C. Thomas, Springfield, Illinois. 